\title{
Study of the wave packet treatment of neutrino oscillation at Daya Bay
}

\author{
Daya Bay Collaboration ${ }^{\mathrm{a},}{ }^{*}$ \\ dayabay.ihep.ac.cn
}

Received: 27 January 2017 / Accepted: 2 June 2017 / Published online: 14 September 2017

(C) The Author(s) 2017. This article is an open access publication

\begin{abstract}
The disappearance of reactor $\bar{v}_{e}$ observed by the Daya Bay experiment is examined in the framework of a model in which the neutrino is described by a wave packet with a relative intrinsic momentum dispersion $\sigma_{\text {rel }}$. Three pairs of nuclear reactors and eight antineutrino detectors, each with good energy resolution, distributed among three experimental halls, supply a high-statistics sample of $\bar{v}_{e}$ acquired at nine different baselines. This provides a unique platform to test the effects which arise from the wave packet treatment of neutrino oscillation. The modified survival probability formula was used to fit Daya Bay data, providing the first experimental limits: $2.38 \times 10^{-17}<\sigma_{\text {rel }}<0.23$. Treating the dimensions of the reactor cores and detectors as constraints, the limits are improved: $10^{-14} \lesssim \sigma_{\text {rel }}<0.23$, and an upper limit of $\sigma_{\text {rel }}<0.20$ (which corresponds to $\sigma_{x} \gtrsim 10^{-11} \mathrm{~cm}$ ) is obtained. All limits correspond to a $95 \%$ C.L. Furthermore, the effect due to the wave packet nature of neutrino oscillation is found to be insignificant for reactor antineutrinos detected by the Daya Bay experiment thus ensuring an unbiased measurement of the oscillation parameters $\sin ^{2} 2 \theta_{13}$ and $\Delta m_{32}^{2}$ within the plane wave model.
\end{abstract}

\section{Contents}

1 Introduction ................. 1

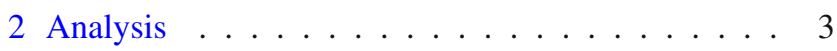

3 Results and discussion . . . . . . . . . . 7

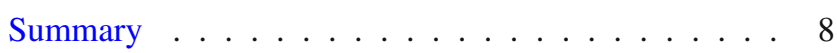

References . . . . . . . . . . . . . 13

* The full list of contributors is displayed at the end of the article.

a e-mail: dmitryvnaumov@gmail.com

\section{Introduction}

1.1 Neutrino oscillation in the plane wave approximation

The neutrino, a light electrically neutral fermion participating in weak interactions, was suggested by Pauli to save the conservation of energy and momentum in nuclear $\beta$-decays. Since then, three flavors of neutrinos $v_{\alpha}=\left(v_{e}, v_{\mu}, v_{\tau}\right)$ were discovered, each produced or detected in association with a corresponding lepton $\ell_{\alpha}=(e, \mu, \tau)$. The neutrinos, which are completely parity-violating in their weak interactions, suggested that the gauge group of the electro-weak sector of the remarkably successful Standard Model (SM) should be built using fermions with left-handed chirality. Given the unique properties of neutrinos, studies of them may reveal a path to physics beyond the SM. In the past, experiments observing solar and atmospheric neutrinos brought increased attention to neutrino physics due to long-standing discrepancies between detection rates and no-oscillation models. Despite an impressive number of proposed solutions to these problems, all were successfully resolved by the hypothesis of neutrino oscillation, first proposed by Pontecorvo [1,2] in the late 1950's. Neutrino oscillation is a phenomenon firmly established in experiment, which has been observed with solar [3-5], atmospheric [6,7], particle accelerator [7,8] and reactor [9-12] neutrinos.

Neutrino oscillation is a quantum phenomenon of quasiperiodic change of neutrino flavor $v_{\alpha} \rightarrow v_{\beta}$ with time. This phenomenon originates in the non-equivalence of neutrino flavor $v_{\alpha}$ and mass $v_{k}=\left(v_{1}, v_{2}, v_{3}\right)$ eigenstates, differences in their masses, and an assumption that the produced and detected neutrino states are coherent superpositions of neutrino mass eigenstates:

$\left|v_{\alpha}(p)\right\rangle=\sum_{k=1}^{3} V_{\alpha k}^{*}\left|v_{k}(p)\right\rangle$, 
where $V_{\alpha k}$ is an element of the unitary PMNS-matrix, named after Pontecorvo, Maki, Nakagawa, Sakata, and $p$ is the momentum of the neutrino. The time evolution of the state in Eq. (1) is expressed as

$\left|v_{\alpha}(t ; p)\right\rangle=\sum_{k=1}^{3} V_{\alpha k}^{*} \mathrm{e}^{-i E_{k} t}\left|v_{k}(p)\right\rangle$,

where $E_{k}=\sqrt{p^{2}+m_{k}^{2}}$. This leads to the oscillatory behavior of the probability to detect a neutrino originally of flavor $\alpha$ as having flavor $\beta$ :

$$
\begin{aligned}
P_{\alpha \beta}(L) & =\left|\left\langle v_{\beta}(p) \mid v_{\alpha}(t ; p)\right\rangle\right|^{2} \\
& =\sum_{k, j=1}^{3} V_{\alpha k}^{*} V_{\beta j}^{*} V_{\beta k} V_{\alpha j} \mathrm{e}^{-i 2 \pi L / L_{k j}^{\mathrm{osc}}},
\end{aligned}
$$

where $L_{k j}^{\text {osc }}=4 \pi p / \Delta m_{k j}^{2}$ is the oscillation length due to the non-zero differences $\Delta m_{k j}^{2}=m_{k}^{2}-m_{j}^{2}$, and time $t$ is approximated by the traveled distance $L$.

The underlying theory, assuming a plane wave approximation, was developed in the middle of the 1970s [13-15]. Although successful in explaining a wide range of neutrino experiments, it is well known that this approximation is not self-consistent, and leads to a number of paradoxes [16,17]. The applicability of the plane wave approximation is discussed in detail in Refs. [16,18-20]. After the first theory was developed, Refs. [21-24] pointed out the necessity of a wave packet treatment of neutrino oscillation.

\subsection{Wave packet treatment of neutrino oscillation}

The wave packet is a coherent superposition of different waves whose momenta are distributed around the most probable value, with a certain "width" or dispersion. Therefore, a wave packet is localized in space-time as well as in energy-momentum space. The wave packet formalism facilitates the resolution of the paradoxes of the plane wave theory, and predicts the existence of a coherence length. The latter arises due to the different group velocities of a pair $v_{k}$ and $v_{j}$, which causes a separation in space over time.

The propagation distance over which a wave (classical or quantum) preserves a certain degree of coherence is known as a coherence length. It is important in many branches of physics. Some examples of classical physics include optics, radio-band systems, holography and telecommunications engineering. Superconductivity, superfluidity and lasers are known as examples of highly coherent quantum systems. Coherence is important in the already available technology of quantum cryptography and in the future technologies of quantum computing. Coherence in neutrino oscillation, being quantum by nature, also exhibits some features of classical systems: two waves $v_{k}$ and $v_{j}$ propagating with different group velocities break the coherence in the quantum state, like in Eq. (1), at distances exceeding the coherence length, similarly to what happens in optics when a wave packet propagates far enough in a medium such that the speed of a wave component with certain frequency depends on the refraction index. The smallness of the difference of neutrino masses suggests that the coherence length of neutrino oscillation is the largest available among all known phenomena.

After the pioneering studies [21-23], the wave packet models of neutrino oscillation were developed in roughly two varieties. The first one relies on a relativistic quantum mechanical (QM) formalism that does not predict the dispersion of the neutrino wave packet in momentum space, such as in Refs. $[18,19,25]$. The second one is based on calculations within quantum field theory (QFT), describing all external particles involved in neutrino production and detection as wave packets while treating neutrinos as virtual particles. The neutrino wave-function is then calculated rather than postulated. The effective momentum dispersion of the neutrino wave function depends on the kinematics of neutrino production and detection and on the momentum dispersions of the external particles, as in Refs. [26-32]. Both approaches predict a number of observable effects, like a quantitative condition on the coherence of mass eigenstates in the production-detection processes, as well as a loss of coherence.

In wave packet models, the intrinsic momentum dispersion $\sigma_{p}$ of the neutrino wave packet is an effective quantity comprising the microscopic momenta dispersions of all particles involved in the production and detection of the neutrino. A non-zero value of $\sigma_{p}$ leads with time to the decoherence in the quantum superposition of massive neutrinos which results in a vanishing oscillation pattern of $v_{\alpha} \rightarrow v_{\beta}$ transitions. In addition, the oscillation pattern is smeared further in the reconstructed energy spectrum due to a non-zero experimental resolution $\delta_{E}$ of the neutrino energy.

Despite considerable progress in building wave packet models, none of these approaches provides a solid quantitative theoretical estimate of $\sigma_{p}$ or of the spatial width $\sigma_{x}=1 / 2 \sigma_{p}$. Theoretical estimates vary by orders of magnitude, associating the dispersion of the neutrino wave packet with various scales; for example, uranium nucleus diameter $\left(\sigma_{x} \simeq 10^{-12} \mathrm{~cm}, \sigma_{p} \simeq 10 \mathrm{MeV}\right)$, atomic or inter-atomic distances $\left(\sigma_{x} \simeq\left(10^{-8}-10^{-7}\right) \mathrm{cm}, \sigma_{p} \simeq\left(10^{3}-10^{2}\right)\right.$ $\mathrm{eV})$, pressure broadening $\left(\sigma_{x} \simeq 10^{-4} \mathrm{~cm}, \sigma_{p} \simeq 0.1 \mathrm{eV}\right)$, etc. While most of the discussions in the current literature does not include calculations of the neutrino wave function from first principles for any type of neutrino experiment, ${ }^{1}$ it

\footnotetext{
1 Recently, a first calculation which consistently treats the full pionneutrino-environment quantum system and calculates the decoherence
} 
also lacks quantitative experimental investigations of decoherence effects in neutrino oscillation inferred from the finite size of the neutrino wave function. ${ }^{2}$

It has been pointed out that a loss of coherence of neutrino mass eigenstates would lead to an event rate smaller than that expected for coherent neutrino states [16]. However, a quantitative study of decoherence effect from the absolute event rate measurements of past reactor experiments [3843 ] is subject to the significant uncertainties in the model predictions of the reactor antineutrino flux.

The day-night asymmetry of solar neutrinos provides an evidence that solar neutrinos come to the Earth in an incoherent mixture [44]. However these data do not provide any quantitative information about the size of a neutrino wave packet because of an averaging over the large volume of the Sun.

One of the motivations of this paper is to provide the first quantitative study of a possible loss of coherence in the quantum state of neutrinos following the wave packet treatment of neutrino oscillations, using data from the Daya Bay Reactor Neutrino Experiment. The second motivation is to demonstrate that the oscillation parameters estimated with the plane wave approximation are unbiased. The oscillation probability formula modified by the wave packet contribution, which is discussed further, has two distinctive features: it depends on $\Delta m_{k j}^{2} / p^{2} \sigma_{\text {rel }}$ via the so-called localization term and on $L \Delta m_{k j}^{2} \sigma_{\text {rel }} / p$ via the term responsible for the loss of coherence with distance, where $\sigma_{\text {rel }}=\sigma_{p} / p$. The large statistics, good energy resolution, and multiple baselines of the Daya Bay experiment make its data valuable in the study of these quantum decoherence effects in neutrino oscillation.

\section{Analysis}

\subsection{Neutrino oscillation in a wave packet model}

Measured energy spectra of $\bar{v}_{e}$ interactions are compared to a prediction using a QM wave packet model of neutrino oscillation which is briefly outlined in what follows. We simplify the consideration by examining a one-dimensional wave packet of the neutrino. ${ }^{3}$ The plane wave state in (1) is replaced by a

Footnote 1 continued

effects for neutrinos produced in two-body decays was published in Ref. [33].

2 Attention to the decoherence phenomena in neutrino oscillation is increasing and the literature discusses possible decoherence effects due to physics beyond the SM like quantum gravity [34-37], differing from the considerations of this paper, which studies the consequences of a self-consistent way to describe neutrino oscillation within the minimally extended Standard Model hosting non-zero mass neutrinos.

${ }^{3}$ While a neutrino travels in the three-dimensional space, the transverse part of its wave function essentially leads to the $1 / L^{2}$ dependence of the flux [45] and does not affect significantly the oscillation pattern. wave packet describing a neutrino produced as flavor $\alpha$ :

$$
\left|\widetilde{v}_{\alpha}\left(p_{P} ; t_{P}, x_{P}\right)\right\rangle=\sum_{k=1}^{3} V_{\alpha k}^{*} \int \frac{d p}{2 \pi} f_{P}(p) \mathrm{e}^{-i \phi_{P}(p)}\left|v_{k}(p)\right\rangle,
$$

with $\phi_{P}(p)=E_{k} t_{P}-p x_{P} . f_{P}(p)$ is the wave function of the neutrino in momentum space and is assumed to be Gaussian:

$f_{P}(p)=\left(\frac{2 \pi}{\sigma_{p P}^{2}}\right)^{\frac{1}{4}} \mathrm{e}^{-\frac{\left(p-p_{P}\right)^{2}}{4 \sigma_{p P}^{2}}}$,

where the subscript $P$ in $f_{P}(p), p_{P}$ and $\sigma_{p P}$ indicates the quantities at production. In configuration space the state in Eq. (4) describes a wave packet with mean coordinate $x_{P}$ at time $t_{P}$. The state in Eq. (4) is normalized to unity. Similarly, a wave packet state at detection $\left|\widetilde{v}_{\beta}\left(p_{D} ; t_{D}, x_{D}\right)\right\rangle$ is defined as the state given by Eq. (4).

A projection of $\left|\widetilde{v}_{\alpha}\left(p_{P} ; t_{P}, x_{P}\right)\right\rangle$ onto $\left\langle\widetilde{v}_{\beta}\left(p_{D} ; t_{D}, x_{D}\right)\right|$ produces the flavor-changing amplitude

$\mathscr{A}_{\alpha \beta}\left(p ; t_{D}-t_{P}, L, \sigma_{p}\right) \equiv\left\langle\widetilde{v}_{\beta}\left(p_{D} ; t_{D}, x_{D}\right) \mid \widetilde{v}_{\alpha}\left(p_{P} ; t_{P}, x_{P}\right)\right\rangle$,

which depends on $L \equiv x_{D}-x_{P}$, time difference $t_{D}-t_{P}$ and on the effective mean neutrino momentum $p$ and momentum dispersion $\sigma_{p}$ comprising the details of production and detection $^{4}$

$p=\frac{p_{P} \sigma_{p D}^{2}+p_{D} \sigma_{p P}^{2}}{\sigma_{p P}^{2}+\sigma_{p D}^{2}}, \quad \frac{1}{\sigma_{p}^{2}}=\frac{1}{\sigma_{p P}^{2}}+\frac{1}{\sigma_{p D}^{2}}$.

The probability $\left|\mathscr{A}_{\alpha \beta}\left(p ; t_{D}-t_{P}, L, \sigma_{p}\right)\right|^{2}$ should be integrated over usually unobservable variables - production time $t_{P}$ (or, equivalently, over $t_{D}-t_{P}$ ) and most probable momentum $p_{P}$ to get an experimentally observable oscillation probability, which does not depend anymore on time $\left(t_{D}-t_{P}\right)$ but does depend on $L$ :

$$
\begin{aligned}
P_{\alpha \beta}(L) & =\int \frac{d t_{P} d p_{P}}{2 \pi}\left|\mathscr{A}_{\alpha \beta}\left(p ; t_{D}-t_{P}, L, \sigma_{p}\right)\right|^{2} \\
& =\sum_{k, j=1}^{3} \frac{V_{\alpha k}^{*} V_{\beta k} V_{\alpha j} V_{\beta j}^{*}}{\sqrt[4]{1+\left(L / L_{k j}^{\mathrm{d}}\right)^{2}}} \mathrm{e}^{-\frac{\left(L / L_{k j}^{\mathrm{coh}}\right)^{2}}{1+\left(L / L_{k j}^{\mathrm{d}}\right)^{2}}-\mathrm{D}_{k j}^{2}} \mathrm{e}^{-i \widetilde{\varphi}_{k j}}
\end{aligned}
$$

where the phase $\widetilde{\varphi}_{k j}$ is the sum of the plane wave phase $\varphi_{k j}=2 \pi L / L_{k j}^{\text {osc }}$ and correction $\varphi_{k j}^{d}$ due to the dispersion of

\footnotetext{
4 The momentum integral in Eq. (6) is calculated by expanding $E_{k}=$ $\sqrt{p^{2}+m_{k}^{2}}$ in a Taylor series up to second order around the effective momentum given by Eq. (7).
} 
the wave packet: $\widetilde{\varphi}_{k j}=\varphi_{k j}+\varphi_{k j}^{d}$, with

$\varphi_{k j}^{\mathrm{d}}=-\frac{L / L_{k j}^{\mathrm{d}}}{1+\left(L / L_{k j}^{\mathrm{d}}\right)^{2}}\left(\frac{L}{L_{k j}^{\mathrm{coh}}}\right)^{2}+\frac{1}{2} \arctan \frac{L}{L_{k j}^{\mathrm{d}}}$.

Oscillation probability formulas similar to Eq. (8) but neglecting wave packet dispersion were obtained in several studies (see, for example, Refs. [18, 29,31,46]). Equation (8) has appeared as a particular case of a more general consideration within QFT with relativistic wave packets [32]. Relativistic invariance suggests that $\sigma_{x} E_{k}$ (and thus $\sigma_{p} / E_{k}$ ) should be a Lorentz invariant $[16,47]$. Up to a typically tiny correction of the order of $m_{k}^{2} / p^{2}, \sigma_{\text {rel }}$ should also be a relativistic invariant, at least when neutrinos remain relativistic. In the QM approach adopted in Eqs. (4)-(8) the only possibility to preserve Lorentz invariance is for $\sigma_{\text {rel }}$ to be a constant. ${ }^{5}$ The probability in Eq. (8) contains three quantities with dimensions of length:

$L_{k j}^{\mathrm{osc}}=\frac{4 \pi p}{\Delta m_{k j}^{2}}, \quad L_{k j}^{\mathrm{coh}}=\frac{L_{k j}^{\mathrm{osc}}}{\sqrt{2} \pi \sigma_{\mathrm{rel}}}$,

$L_{k j}^{\mathrm{d}}=\frac{L_{k j}^{\mathrm{coh}}}{2 \sqrt{2} \sigma_{\mathrm{rel}}}$,

where $L_{k j}^{\text {osc }}$ is the usual oscillation length of a pair of neutrino states $\left|v_{k}\right\rangle$ and $\left|v_{j}\right\rangle, L_{k j}^{\text {coh }}$ is interpreted as the neutrino coherence length, i.e. the distance at which the interference of neutrino mass eigenstates vanishes, and finally $L_{k j}^{\mathrm{d}}$ is the dispersion length, i.e. a distance at which the wave packet is doubled in its spatial dimension due to the dispersion of waves moving with different velocities. The term

$\mathrm{D}_{k j}^{2}=\frac{1}{2}\left(\frac{\Delta m_{k j}^{2}}{4 p^{2} \sigma_{\text {rel }}}\right)^{2}=\frac{1}{4}\left(\frac{\Delta m_{k j}^{2}}{\sigma_{m^{2}}}\right)^{2}=\left(\frac{\sqrt{2} \pi \sigma_{x}}{L_{k j}^{\text {osc }}}\right)^{2}$

suppresses the coherence of massive neutrino states $\left|v_{k}\right\rangle$ and $\left|v_{j}\right\rangle$ if $\Delta m_{k j}^{2} \gg \sigma_{m^{2}}$, where $\sigma_{m^{2}}=2 \sqrt{2} p \sigma_{p}$ could be interpreted as an uncertainty in the neutrino mass squared [22]. $\mathrm{D}_{k j}^{2}$ can be seen from another perspective as the localization term suppressing the oscillation if $\sqrt{2} \pi \sigma_{x} \gg L_{k j}^{\text {osc }}$, where $\sigma_{x}=\left(2 \sigma_{p}\right)^{-1}$ is the width of neutrino wave packet in the configuration space.

\footnotetext{
5 Since the QFT approach considers both neutrino production and detection one finds that $\sigma_{\text {rel }}$, being a relativistic invariant, is actually a function of kinematic variables involved in the production and detection processes as well as of momentum dispersions of wave packets describing all involved particles [48]. Therefore, in comparing the QM and QFT approaches, we may treat the QM $\sigma_{\text {rel }}$ as that of the QFT approach averaged over the kinematic variables of all external wave packets involved in neutrino production and detection.
}

It is worth mentioning that terms in Eq. (8) which correspond to the interference of $v_{k}$ and $v_{j}$ states also get suppressed by the denominator $\sqrt[4]{1+\left(L / L_{k j}^{\mathrm{d}}\right)^{2}}$ and vanish for both limits $\sigma_{p} \rightarrow 0$ and $\sigma_{p} \rightarrow \infty$, reducing the oscillation probability in Eq. (8) to the non-coherent sum

$P_{\alpha \beta}=\sum_{k}\left|V_{\alpha k}\right|^{2}\left|V_{\beta k}\right|^{2}$

which does not depend on energy and distance. The oscillation probability in Eq. (8b) is not reduced to the plane wave formula in Eq. (3) in the limit $\sigma_{p} \rightarrow 0$ because of the integration over an unobservable production time $t_{P}$ in Eq. (8a) which is necessary in a self-consistent consideration. Let us observe, that a time average of Eq. (3) also leads to noncoherent formula in Eq. (12).

It is always possible for the given values of $p$ and $L$ to identify the domain of $\sigma_{p}$ where Eqs. (3) and (8b) are numerically almost identical to each other (see Sect. 2.2).

For the $\bar{v}_{e}$ at Daya Bay, $1-P_{\mathrm{ee}}$ is expressed as

$$
\begin{aligned}
& \frac{1}{2} \sin ^{2} 2 \theta_{12} \cos ^{4} \theta_{13} \\
& \times\left(1-\frac{\exp \left[-\frac{\left(L / L_{21}^{\mathrm{coh}}\right)^{2}}{1+\left(L / L_{21}^{\mathrm{d}}\right)^{2}}-D_{21}^{2}\right]}{\sqrt[4]{1+\left(L / L_{21}^{\mathrm{d}}\right)^{2}}} \cos \left(\varphi_{21}+\varphi_{21}^{\mathrm{d}}\right)\right) \\
& +\frac{1}{2} \cos ^{2} \theta_{12} \sin ^{2} 2 \theta_{13} \\
& \times\left(1-\frac{\exp \left[-\frac{\left(L / L_{31}^{\mathrm{coh}}\right)^{2}}{1+\left(L / L_{31}^{\mathrm{d}}\right)^{2}}-D_{31}^{2}\right]}{\sqrt[4]{1+\left(L / L_{31}^{\mathrm{d}}\right)^{2}}} \cos \left(\varphi_{31}+\varphi_{31}^{\mathrm{d}}\right)\right) \\
& +\frac{1}{2} \sin ^{2} \theta_{12} \sin ^{2} 2 \theta_{13} \\
& \times\left(1-\frac{\exp \left[-\frac{\left(L / L_{32}^{\mathrm{coh}}\right)^{2}}{1+\left(L / L_{32}^{\mathrm{d}}\right)^{2}}-D_{32}^{2}\right]}{\sqrt[4]{1+\left(L / L_{32}^{\mathrm{d}}\right)^{2}}} \cos \left(\varphi_{32}+\varphi_{32}^{\mathrm{d}}\right)\right) .
\end{aligned}
$$

2.2 Sensitivity of Daya Bay experiment to neutrino wave packet

The Daya Bay experiment is composed of two near underground experimental halls (EH1 and EH2) and one far underground hall (EH3). Each of the experimental halls hosts identically designed antineutrino detectors (ADs). EH1 and $\mathrm{EH} 2$ contain two ADs each, while EH3 contains four ADs. Electron antineutrinos are produced in three pairs of nuclear reactors via $\beta$ decays of neutron-rich daughters of the fis- 
Table 1 The number of IBD candidates and mean distances of the three experimental halls to the pairs of reactor cores

\begin{tabular}{lllcc}
\hline \multirow{2}{*}{ Halls } & IBD candidates & \multicolumn{3}{c}{ Mean distance, $\mathrm{m}$} \\
\cline { 3 - 5 } & & Daya Bay & Ling Ao & Ling Ao II \\
\hline EH1 & 613,813 & 365 & 860 & 1310 \\
EH2 & 477,144 & 1348 & 481 & 529 \\
EH3 & 150,255 & 1909 & 1537 & 1542 \\
\hline
\end{tabular}

sion isotopes ${ }^{235} \mathrm{U},{ }^{238} \mathrm{U},{ }^{239} \mathrm{Pu}$ and ${ }^{241} \mathrm{Pu}$, and detected via the inverse $\beta$ decay (IBD). The coincidence of the prompt $\left(e^{+}\right.$ionization and annihilation) and delayed ( $n$ capture on Gd) signals efficiently suppresses the backgrounds, which amounted to less than $2 \%(5 \%)$ of the IBD candidates in the near (far) halls [49]. The Gd-doped liquid scintillator target is a cylinder of three meters in both height and diameter. The detectors have a light yield of about 165 photoelectrons/ $\mathrm{MeV}$ and a reconstructed energy resolution $\delta_{E} / E \approx 8 \%$ at $1 \mathrm{MeV}$ of deposited energy in the scintillator. More details on the experimental setup are contained in Refs. [49-52].

The studies in this paper are based on data acquired in the 6-AD period when there were two ADs in $\mathrm{EH} 1$, one $\mathrm{AD}$ in $\mathrm{EH} 2$ and $3 \mathrm{ADs}$ in $\mathrm{EH} 3$, with the addition of the 8-AD period from October 2012 to November 2013, a total of 621 days. The number of IBD candidates used in this analysis, and the mean baselines of the three experimental halls to each pair of reactor cores, are summarized in Table 1. The expected numbers of IBD events are convolutions of the reactor-to-target expectation with the detectorresponse function. The reactor-to-target expectation takes into account the antineutrino fluxes from each reactor core including non-equilibrium and spent nuclear fuel corrections, first order in $1 / m_{p}$ ( $m_{p}=$ proton mass) IBD crosssection accounting for the positron emission angle [53], and the oscillation survival probability $P_{\text {ee }}$ given by Eq. (3) for the plane wave model and by Eq. (8) for the wave packet model. The detector response-function accounts for energy loss in the inner acrylic vessel, liquid scintillator and electronics non-linearity and energy resolution $\delta_{E}$.

One can meet claims in literature that the smallest among $\sigma_{p}$ and $\delta_{E}$ determines the decoherence effects in neutrino oscillations. In what follows, we provide some qualitative and analytical arguments showing the actual interplay of intrinsic momentum dispersion $\sigma_{p}$ of neutrino wave packet and $\delta_{E}$. The latter is sometimes erroneously considered as an upper extreme value of $\sigma_{p}$. The width $\left(\Gamma \simeq \sigma_{p}\right)$ of a hadronic resonance which is typically much larger than an experimental energy resolution $\delta_{E}$ provides a well-known counter-example, illustrating that $\sigma_{p}$ could be much larger than $\delta_{E}$.
For relatively large values of $\sigma_{p} \simeq \delta_{E}$, the effects of these two parameters on the observed energy spectra might appear similar, however they are distinct. First, they have different physical origins: while $\sigma_{p}$ is governed by the most localized particle in the production and detection of the neutrino, $\delta_{E}$ is determined by the energy depositions of the final state particles in the detector, the amount and efficiency of detection devices used to observe such depositions. In particular, considering a liquid scintillator detector surrounded by a number of PMTs as an example, one could hypothesize modifications in the number of PMTs, their efficiencies or even in the light yield. Such variations would modify the energy resolution $\delta_{E}$ correspondingly, leaving intact the microscopic processes determining $\sigma_{p}$ and, respectively, the number of neutrino interactions in the detector. Second, these effects can also be distinguished from their order of occurrence since the microscopic processes used in the energy estimation occur later in time with respect to the neutrino interaction in the detector. Third, their effects are not identical. In particular, as described in Sec. 2.1, the limit $\sigma_{p} \rightarrow 0$ leads to the decoherence of neutrino oscillation in contrast to the impact of energy resolution which does not lead to any smearing in the reconstructed energy spectrum in the limit $\delta_{E} \rightarrow 0$.

In order to illustrate analytically an interplay of $\sigma_{p}$ and $\delta_{E}$, let us consider the exponential in the oscillation probability in Eq. (8) convolved with a Gaussian energy resolution, as a function of the reconstructed energy $E_{\mathrm{vis}}$, assuming $\delta_{E} \ll p$, infinite dispersion length $L^{\mathrm{d}}$, neglecting the $D^{2}$ term, and suppressing mass eigenstate indices for the sake of compactness $^{6}$ :

$$
\begin{aligned}
& \frac{1}{\sqrt{2 \pi} \delta_{E}} \int d p \exp \left(-i 2 \pi L / L^{\mathrm{osc}}-\left(L / L^{\mathrm{coh}}\right)^{2}\right. \\
& \left.\quad-\left(p-E_{\mathrm{vis}}\right)^{2} / 2 \delta_{E}^{2}\right) \\
& \simeq \exp \left(-i 2 \pi L / L_{\mathrm{rec}}^{\mathrm{osc}}-\left(L / L_{\mathrm{eff}}^{\mathrm{coh}}\right)^{2}\right),
\end{aligned}
$$

where $L^{\mathrm{osc}}$ and $L^{\mathrm{coh}}$ are given by Eq. (10) and the effective coherence length comprises both the intrinsic $\sigma_{p}$ and detector resolution $\delta_{E}$ :

$$
\left(\frac{1}{L_{\mathrm{eff}}^{\mathrm{coh}}}\right)^{2}=\left(\frac{1}{L_{\mathrm{rec}}^{\mathrm{coh}}}\right)^{2}+\left(\frac{1}{L_{\mathrm{det}}^{\mathrm{coh}}}\right)^{2},
$$

where $L_{\mathrm{rec}}^{\mathrm{osc}}$ and $L_{\mathrm{rec}}^{\mathrm{coh}}$ are given by $L^{\mathrm{osc}}$ and $L^{\mathrm{coh}}$ replacing $p$ with $E_{\mathrm{vis}}$, and $L_{\mathrm{det}}^{\mathrm{coh}}$ is given by $L_{\mathrm{rec}}^{\mathrm{coh}}$, replacing $\sigma_{p}$ with $\delta_{E}$. The interplay of $\sigma_{p}$ and $\delta_{E}$ is illustrated by the effective coherence length $L_{\text {eff }}^{\text {coh }}$, which is dominantly determined by the smallest among $L_{\mathrm{rec}}^{\text {coh }}$ and $L_{\mathrm{det}}^{\mathrm{coh}}$, or by the largest among $\sigma_{p}$ and $\delta_{E}$. Therefore, the effective energy dispersion $\sigma_{p}^{\text {eff }}$ is determined by $\left(\sigma_{p}^{\text {eff }}\right)^{2}=\sigma_{p}^{2}+\delta_{E}^{2}$.

\footnotetext{
6 The actual implementation of the detector effects in this analysis was performed numerically without approximations.
} 
The following provides simple numerical estimates of Daya Bay sensitivity to wave packet effects on neutrino oscillations.

For a typical momentum of $p=4 \mathrm{MeV}$ of detected reactor $\bar{v}_{e}$, the oscillation would be suppressed for two distinctive domains of $\sigma_{\text {rel }}$. The domain $\sigma_{\text {rel }} \gtrsim O(0.1)$ corresponds to significant contributions from $L$-dependent interferencesuppressing terms and corrections to the oscillation phase $\varphi_{32}^{d}$ in Eq. (8), while the $D_{k j}^{2}$ term is negligibly small. For example, at $L=L_{32}^{\mathrm{osc}} / 2$ the exponential suppression reaches its maximum $\mathrm{e}^{-\pi / 8}$ at $\sigma_{\text {rel }}=1 / \sqrt{2 \pi} \simeq 0.4$. Correspondingly, the coherence and dispersion lengths read $L_{32}^{\text {coh }} \simeq 2.2$ $\mathrm{km}$ and $L_{32}^{\mathrm{d}} \simeq 2 \mathrm{~km}$. At larger values of $\sigma_{\text {rel }}$ and at a fixed distance the spatial dispersion of neutrino wave packets partially compensates the loss of coherence due to the spatial separation of $v_{k}$ and $v_{j}$.

The domain $\sigma_{\text {rel }} \lesssim O\left(2.8 \times 10^{-17}\right)$ corresponds to $D_{32}^{2} \gtrsim 1$, which is significant in suppressing the interference in Eq. (8) through the $L$-independent term, while the $L$-dependent terms are negligibly small. Thus, the region of $O\left(2.8 \times 10^{-17}\right) \ll \sigma_{\text {rel }} \ll O(0.1)$ is where the wave packet impact on neutrino oscillation is negligible for the Daya Bay experiment.

For illustrative purposes Fig. 1 shows the ratio of the observed to expected numbers of IBD events assuming no oscillation using the data collected at the near and far experimental halls as a function of reconstructed visible energy $E_{\text {vis. }}$. Figure 1 also shows the expected ratio for neutrino oscillation with the plane wave and wave packet models with $\sigma_{\text {rel }}$ of 0.33 and $8 \times 10^{-17}$ as examples.

Both model expectations are shown with the oscillation parameters fixed to their best-fit values within the plane wave model. ${ }^{7}$ For this set of parameters, the wave packet models with $\sigma_{\text {rel }}=0.33$ and with $\sigma_{\text {rel }}=8 \times 10^{-17}$ are inconsistent with the data by about five standard deviations, thus motivating the chosen values of $\sigma_{\text {rel }}$. The two panels illustrate how the visible energy spectra are modified in the near and far halls depending on the intrinsic dispersion of the neutrino wave packet. Remarkably, most changes in the energy spectra due to $\sigma_{\text {rel }}$ are in opposite directions for near and far halls, which can be explained qualitatively as follows. As mentioned above, the extremes $\sigma_{p} \rightarrow 0$ and $\sigma_{p} \rightarrow \infty$ would yield fully decoherent neutrinos with the oscillation probability given by Eq. (12). Antineutrinos detected at the near halls experience a relatively small oscillation in the plane wave approach. The values of $\sigma_{\text {rel }}$ selected for Fig. 1 make the $\bar{v}_{e}$ partially decoherent and $P_{\text {ee }}$ tend towards Eq. (12), predicting a smaller number of surviving $\bar{v}_{e}$ as compared to the plane wave formula. The distance at which the far detectors

7 The following values of the oscillation parameters were used in Fig. 1: $\Delta m_{21}^{2}=7.53 \times 10^{-5} \mathrm{eV}^{2}, \Delta m_{32}^{2}=2.45 \times 10^{-3} \mathrm{eV}^{2}, \sin ^{2} 2 \theta_{12}=$ $0.846, \sin ^{2} 2 \theta_{13}=0.0852$.

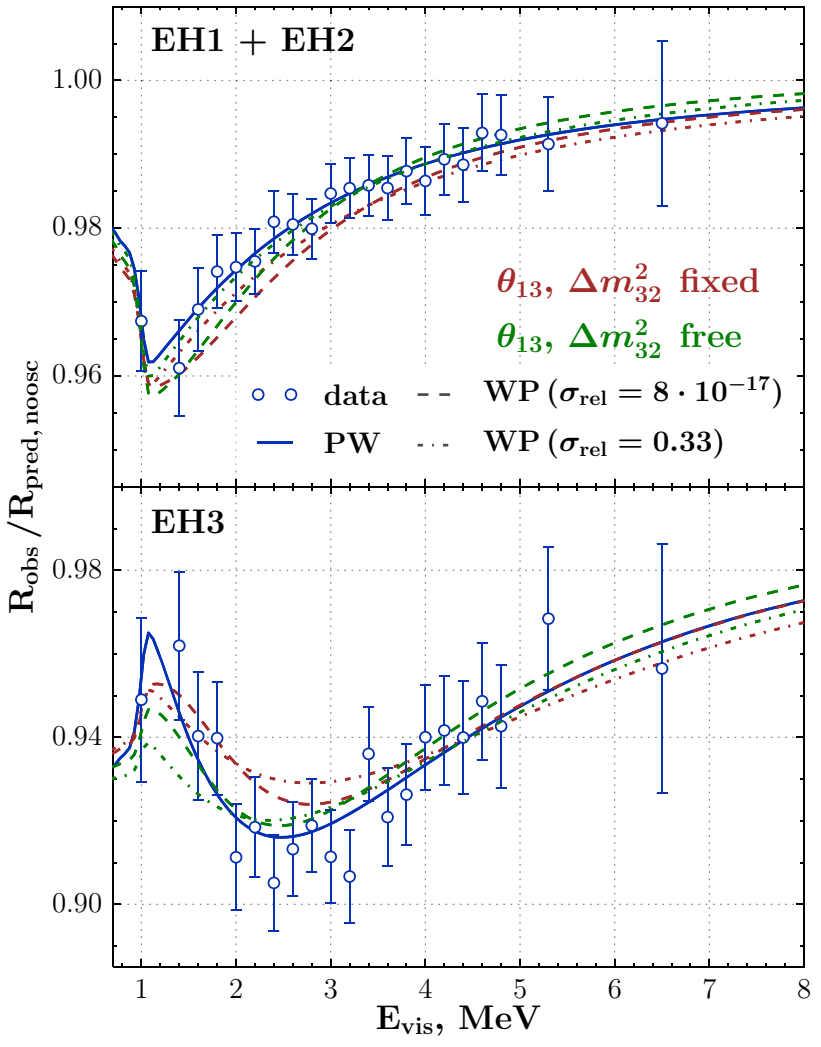

Fig. 1 Ratios of the observed to expected numbers of IBD events in the absence of oscillation as a function of reconstructed visible energy $E_{\mathrm{vis}}$. The data are grouped by near $(\mathrm{EH} 1+\mathrm{EH} 2)$ and far $(\mathrm{EH} 3)$ halls, displayed in the upper and in the bottom panels respectively, with the error bars representing the statistical uncertainties. Superimposed solid lines are ratios assuming neutrino oscillations within the plane wave model (PW) with the best-fit values of $\sin ^{2} 2 \theta_{13}$ and $\Delta m_{32}^{2}$ obtained with the plane wave model. The ratios using the wave-packet model (WP) assume $\sigma_{\text {rel }}=0.33$ (dashed line) and $\sigma_{\text {rel }}=8 \times 10^{-17}$ (dotdashed line), as two examples. The green lines correspond to the wave packet model ratios assuming the best-fit values of $\sin ^{2} 2 \theta_{13}$ and $\Delta m_{32}^{2}$ obtained with the plane wave model and thus, inconsistent with the data by about five standard deviations. The red lines correspond to the wave packet model ratios assuming the best-fit values of $\sin ^{2} 2 \theta_{13}$ and $\Delta m_{32}^{2}$ obtained within the wave packet model, yielding a much better agreement with the data. All ratios enter the region below $2 m_{e}$, which corresponds to the IBD threshold, because of detector response effects like energy reconstruction and absorption in the inner acrylic vessel (see details in Refs. [49,52])

of the Daya Bay experiment are placed is tuned to observe the maximal oscillation effect due to $\Delta m_{32}^{2}$. Partial decoherence of the $\bar{v}_{e}$ tends to reduce the oscillation, thus predicting a larger number of survived $\bar{v}_{e}$ with respect to the plane wave formula. This feature of Daya Bay provides additional sensitivity to the decoherence effects and makes such a study less sensitive to the predicted reactor $\bar{v}_{e}$ spectrum.

The data can be reasonably well described by

$\Delta m_{32}^{2}=2.17 \times 10^{-3} \mathrm{eV}^{2}, \quad \sin ^{2} 2 \theta_{13}=0.102$,

$\sigma_{\text {rel }}=8 \times 10^{-17}, \quad \chi^{2} /$ ndf $=246.8 /(256-4)$, 
and by

$\Delta m_{32}^{2}=2.16 \times 10^{-3} \mathrm{eV}^{2}, \quad \sin ^{2} 2 \theta_{13}=0.097$,

$\sigma_{\text {rel }}=0.33, \quad \chi^{2} / \mathrm{ndf}=253.8 /(256-4)$.

These results demonstrate that one could obtain reasonable fits of the data within the wave packet model with certain values of $\sigma_{\text {rel }}$ and yield best-fit values of the oscillation parameters which differ from the corresponding best-fit values with the plane wave model, assuming normal mass hierarchy ${ }^{8}$ :

$$
\begin{aligned}
& \Delta m_{32}^{2}=2.45 \times 10^{-3} \mathrm{eV}^{2}, \quad \sin ^{2} 2 \theta_{13}=0.0852, \\
& \chi^{2} / \text { ndf }=245.9 /(256-3) .
\end{aligned}
$$

However, Eqs. $(16,17)$ do not correspond to the global minimum of the $\chi^{2}$ discussed below because $\sigma_{\text {rel }}$ was fixed to two arbitrary values for illustrative purposes. In order to find the global minimum we performed a detailed statistical analysis of the allowed region of $\sigma_{\text {rel }}$.

\subsection{Statistical framework}

As the goodness-of-fit measure we use $\chi^{2}(\boldsymbol{\eta})=(\mathbf{d}-$ $\mathbf{t}(\boldsymbol{\eta}))^{T} V^{-1}(\mathbf{d}-\mathbf{t}(\boldsymbol{\eta}))$, where $\mathbf{d}$ is a data vector containing detected numbers of IBD candidates in energy bins and in different detectors, while $\mathbf{t}(\boldsymbol{\eta})$ is the corresponding theoretical model vector which depends on constrained and unconstrained parameters $\eta$. All constraints of the model as well as expected fluctuations in the number of IBD events are encompassed in the covariance matrix $V$. The model vector $\mathbf{t}(\boldsymbol{\eta})$ comprises expected numbers of IBD and background events. All constrained parameters (or systematic uncertainties) relevant for the Daya Bay oscillation analyses were taken into account in this analysis. These are mainly associated with the reactor antineutrino flux, background predictions and the detector response modeling. The uncertainty of the detector response is dominant. Details can be found in Refs. [49,52].

The analysis was done with four unconstrained parameters $\sigma_{\text {rel }}, \Delta m_{32}^{2}, \sin ^{2} 2 \theta_{13}$ and reactor flux normalization $N$. The confidence regions are produced by means of two statistical methods: the conventional fixed-level $\Delta \chi^{2}$ analysis and the Feldman-Cousins method [54]. The marginalized $\Delta \chi^{2}$ statistic is

$\Delta \chi^{2}\left(\boldsymbol{\eta}^{\prime}\right)=\min _{\boldsymbol{\eta} \backslash \boldsymbol{\eta}^{\prime}} \chi^{2}(\boldsymbol{\eta})-\min _{\boldsymbol{\eta}} \chi^{2}(\boldsymbol{\eta})$

where $\eta=\left(\sigma_{\text {rel }}, \Delta m_{32}^{2}, \sin ^{2} 2 \theta_{13}, N\right)$ and $\eta^{\prime}$ is its subspace with parameters of interest $\left(\boldsymbol{\eta}^{\prime}=\sigma_{\text {rel }}\right.$ for one dimensional interval, and $\boldsymbol{\eta}^{\prime}=\left(\sigma_{\text {rel }}, \Delta m_{32}^{2}\right)$ or $\boldsymbol{\eta}^{\prime}=\left(\sigma_{\text {rel }}, \sin ^{2} 2 \theta_{13}\right)$ for

\footnotetext{
8 The best-fit values of the oscillation parameters $\sin ^{2} 2 \theta_{13}$ and $\Delta m_{32}^{2}$ are different from our previous publication [49] because of a different implementation of systematic uncertainties and another choice of $E_{\mathrm{vis}}$ binning.
}

two dimensional regions), and both are used to determine the $p$-value of the observed dataset and the model.

The closed interval corresponding to the $100 \times(1-\alpha) \%$ confidence level (C.L.) is constructed for both the fixed-level $\Delta \chi^{2}$ analysis and the Feldman-Cousins method as the region of $\boldsymbol{\eta}^{\prime}$ which satisfies:

$\Delta \chi^{2}\left(\boldsymbol{\eta}^{\prime}\right)<\Delta \chi_{1-\alpha}^{2}$,

where $\Delta \chi_{1-\alpha}^{2}$ is the $(1-\alpha)$-th quantile of the statistic in Eq. (19). The tabulated values of the quantile $\chi_{n ; 1-\alpha}^{2}$ of the $\chi_{n}^{2}$ distribution with $n$ degrees of freedom ( $n=1,2$ for one and two dimensional confidence regions) were used for the fixed-level $\Delta \chi^{2}$ analysis. Toy Monte Carlo sampling was used to determine $\Delta \chi_{1-\alpha}^{2}$ of the statistic in Eq. (19) with the Feldman-Cousins method.

An open confidence interval can be constructed if neutrinos are assumed to be produced and detected coherently, which is equivalent to assuming $\sigma_{\text {rel }} \gg 10^{-16}$. In this case, instead of using Eq. (19), an upper bound on $\sigma_{\text {rel }}$ can be computed using the modified statistic [55]

$\Delta \chi_{\text {up }}^{2}\left(\sigma_{\text {rel }}\right)= \begin{cases}\Delta \chi^{2}\left(\sigma_{\text {rel }}\right) & \text { if } \hat{\sigma}_{\text {rel }}<\sigma_{\text {rel }} \\ 0 & \text { if } \hat{\sigma}_{\text {rel }}>\sigma_{\text {rel }}\end{cases}$

with $\hat{\sigma}_{\text {rel }}$ representing the best-fit value. In the fixed-level $\Delta \chi^{2}$ analysis the $100 \times(1-\alpha) \%$ C.L. upper limit is given by:

$\Delta \chi^{2}\left(\sigma_{\text {rel }}\right) \leq \chi_{1 ; 1-2 \alpha}^{2}$.

For example, in order to set a 95\% C.L. upper limit, the quantile $\chi_{1 ; 0.9}^{2}=2.71$ was used. The Feldman-Cousins method automatically produces the proper interval using the interval construction in Eq. (20).

\section{Results and discussion}

Figure 2 displays the allowed regions in $\left(\Delta m_{32}^{2}, \sigma_{\text {rel }}\right)$ and $\left(\sin ^{2} 2 \theta_{13}, \sigma_{\text {rel }}\right)$ obtained with both the fixed-level $\Delta \chi^{2}$ and the Feldman-Cousins methods, which are found to be consistent. For the values of $\sigma_{\text {rel }} \lesssim 10^{-16}$ the decoherence effects lead to strong correlations between $\Delta m_{32}^{2}, \sin ^{2} 2 \theta_{13}$ and $\sigma_{\text {rel }}$, yielding smaller values of $\Delta m_{32}^{2}$ and larger values of $\sin ^{2} 2 \theta_{13}$. These correlations are expected taking into account the explicit form of $1-P_{\mathrm{ee}}(L)$ in Eq. (13). The coefficients of $\sigma_{\text {rel }}$ correlation with $\sin ^{2} 2 \theta_{13}$ and $\Delta m_{32}^{2}$ are found to be -0.98 and 0.96 respectively. For $\sigma_{\text {rel }} \gtrsim O(0.1)$, these correlations are found to be significantly weaker. The absolute values of the corresponding correlation coefficients are smaller than $10^{-5}$. 


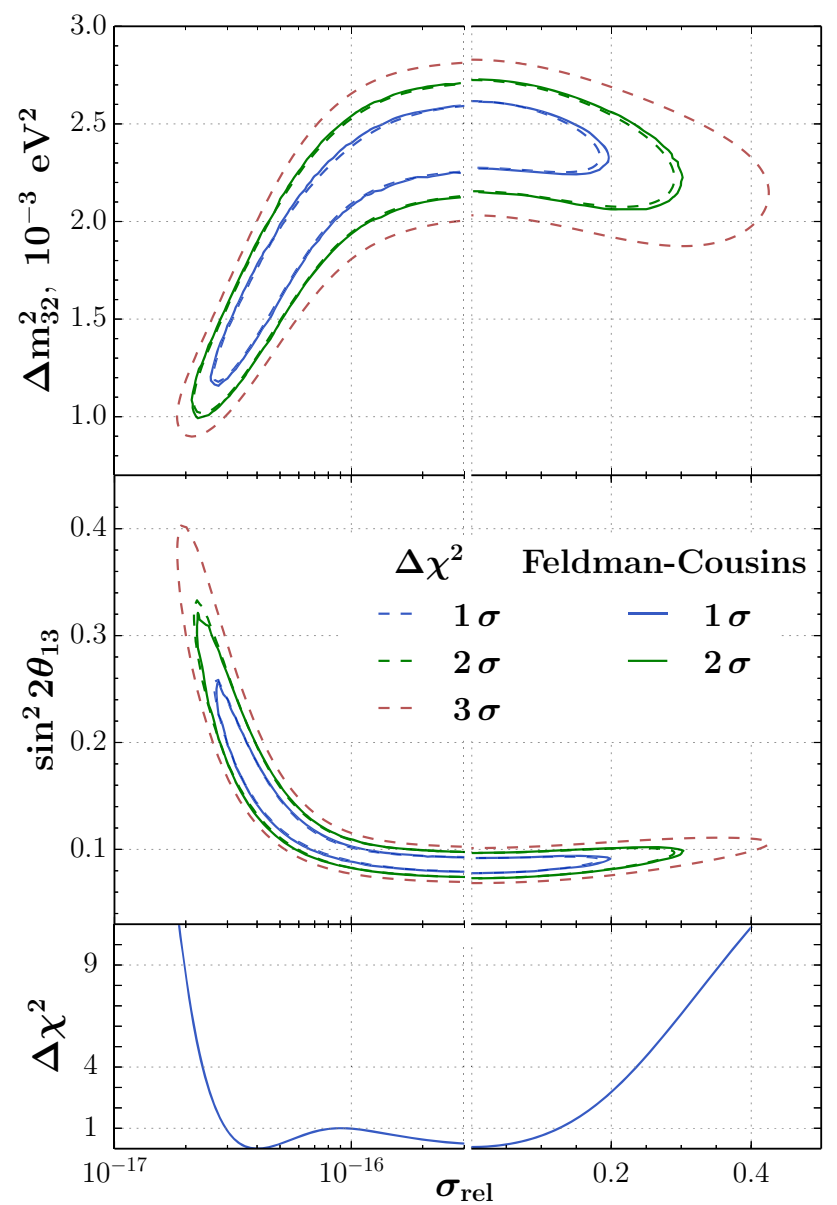

Fig. 2 Allowed regions of $\left(\Delta m_{32}^{2}, \sigma_{\text {rel }}\right)($ top $)$ and of $\left(\sin ^{2} 2 \theta_{13}, \sigma_{\text {rel }}\right)$ (middle) parameters obtained with fixed-level $\Delta \chi^{2}$ (contours corresponding to $1 \sigma, 2 \sigma, 3 \sigma$ C.L., dashed lines) and within the FeldmanCousins (contours corresponding to $1 \sigma, 2 \sigma$ C.L., solid lines) methods. Bottom panel shows the marginalized $\Delta \chi^{2}\left(\sigma_{\text {rel }}\right)$ statistic given by (19) vs $\sigma_{\text {rel }}$. Note the break in the abscissa and the change from a logarithmic to linear scale

The best-fit point corresponds to

$\Delta m_{32}^{2}=1.59 \times 10^{-3} \mathrm{eV}^{2}, \quad \sin ^{2} 2 \theta_{13}=0.160$,

$\sigma_{\text {rel }}=4.0 \times 10^{-17}, \quad \chi^{2} / \mathrm{ndf}=245.9 /(256-4)$,

with the p-value 0.596 which is smaller than the p-value 0.614 with the plane wave model given by Eq. (18). The allowed region for $\sigma_{\text {rel }}$ at a $95 \%$ C.L. reads:

$2.38 \times 10^{-17}<\sigma_{\text {rel }}<0.23$.

Taking the average momentum $p=4 \mathrm{MeV}$ of detected reactor $\bar{v}_{e}$, the interval in Eq. (24) can be translated to $10^{-11} \mathrm{~cm} \lesssim \sigma_{x} \lesssim 1 \mathrm{~km}$. The upper bound of Eq. (24) ensures that the coherence is preserved during at least almost two oscillation half-cycles: $L_{32}^{\text {coh }}>1.94 L_{32}^{\text {osc }} / 2$ while the dispersion length is larger than almost three oscillation halfcycles: $L_{32}^{\mathrm{d}}>2.96 L_{32}^{\mathrm{osc}} / 2$.
The lower limit of Eq. (24) $\left(\sigma_{x} \lesssim 1 \mathrm{~km}\right)$ obtained by constraining the $D_{k j}^{2}$ term is much weaker than an obvious constraint of $\sigma_{x} \lesssim 2 \mathrm{~m}$ which follows from the consideration that the $\sigma_{x}$ (which equals $1 / 2 \sigma_{p}$ ) of $\bar{v}_{e}$ wave packets detected by the Daya Bay Experiment does not exceed the dimensions of the reactor cores and detectors. Taking this constraint into account, $\sigma_{p} \gtrsim 5 \times 10^{-8} \mathrm{eV}$, which for the average momentum $p=4 \mathrm{MeV}$, translates into $\sigma_{\text {rel }} \gtrsim 10^{-14}$. Such a $\sigma_{\text {rel }}$ corresponds to the regime where $D_{k j}^{2} \ll 1$ and the localization term can be safely neglected, which allows us, using the modified statistic for an open interval in Eq. (21), to put an upper limit of:

$\sigma_{\text {rel }}<0.20$, at a $95 \%$ C.L.

Future reactor experiments at baselines of approximately 50 $\mathrm{km}$ such as JUNO [56] and RENO-50 [57] would be able to improve the upper limit on $\sigma_{\text {rel }}$ by more than an order of magnitude due to about 20 oscillation cycles to be detected and unprecedented resolution of visible energy of $\delta_{E} / E \simeq$ $3 \% / \sqrt{E}$. We estimate the following sensitivity of JUNO: $3.8 \times 10^{-17}<\sigma_{\text {rel }}<0.01$ at a $95 \%$ C.L. The lower limit cannot be improved by JUNO because of smaller statistics of expected $\bar{v}_{e}$ interactions with respect to Daya Bay and independence of $D_{k j}^{2}$ on the baseline.

\section{Summary}

We performed a search for the footprint of the neutrino wave packet which should show itself through specific modifications of the neutrino oscillation probability. The reported analysis of the Daya Bay data provides, for the first time, an allowed interval of the intrinsic relative dispersion of neutrino momentum $2.38 \times 10^{-17}<\sigma_{\text {rel }}<0.23$. Taking into account the actual dimensions of the reactor cores and detectors, we find that the lower limit $\sigma_{\text {rel }}>10^{-14}$ corresponds to the regime when the localization term is vanishing, thus allowing us to put an upper limit: $\sigma_{\text {rel }}<0.20$ at a $95 \%$ C.L. This upper limit of $\sigma_{\text {rel }}$ implies that $\sigma_{x} \gtrsim 10^{-11} \mathrm{~cm}$ exceeds size of any nucleus thus excluding a theoretical possibility of neutrino wave function to be formed at nuclear scales.

The current limits are dominated by statistics. With three years of additional data the upper limit on $\sigma_{\text {rel }}$ is expected to be improved by about $30 \%$. The allowed decoherence effect due to the wave packet nature of neutrino oscillation is found to be insignificant for reactor antineutrinos detected by the Daya Bay experiment thus ensuring an unbiased measurement of the oscillation parameters $\sin ^{2} 2 \theta_{13}$ and $\Delta m_{32}^{2}$ within the plane wave model. 
Acknowledgements Daya Bay is supported in part by the Ministry of Science and Technology of China, the U.S. Department of Energy, the Chinese Academy of Sciences, the CAS Center for Excellence in Particle Physics, the National Natural Science Foundation of China, the Guangdong provincial government, the Shenzhen municipal government, the China General Nuclear Power Group, Key Laboratory of Particle and Radiation Imaging (Tsinghua University), the Ministry of Education, Key Laboratory of Particle Physics and Particle Irradiation (Shandong University), the Ministry of Education, Shanghai Laboratory for Particle Physics and Cosmology, the Research Grants Council of the Hong Kong Special Administrative Region of China, the University Development Fund of The University of Hong Kong, the MOE program for Research of Excellence at National Taiwan University, National Chiao-Tung University, and NSC fund support from Taiwan, the U.S. National Science Foundation, the Alfred P. Sloan Foundation, the Ministry of Education, Youth, and Sports of the Czech Republic, the Joint Institute of Nuclear Research in Dubna, Russia, the RFBR research program, the National Commission of Scientific and Technological Research of Chile, and the Tsinghua University Initiative Scientific Research Program. We acknowledge Yellow River Engineering Consulting Co., Ltd., and China Railway 15th Bureau Group Co., Ltd., for building the underground laboratory. We are grateful for the ongoing cooperation from the China General Nuclear Power Group and China Light and Power Company.

Author list F. P. An: Institute of Modern Physics, East China University of Science and Technology, Shanghai.

A. B. Balantekin: University of Wisconsin, Madison, WI 53706, USA.

H. R. Band: Department of Physics, Yale University, New Haven, CT 06520, USA.

M. Bishai: Brookhaven National Laboratory, Upton, NY 11973, USA.

S. Blyth: Department of Physics, National Taiwan University, Taipei; National United University, Miao-Li.

D. Cao: Nanjing University, Nanjing.

G. F. Cao: Institute of High Energy Physics, Beijing.

J. Cao: Institute of High Energy Physics, Beijing.

W. R. Cen: Institute of High Energy Physics, Beijing.

Y. L. Chan: Chinese University of Hong Kong, Hong Kong.

J. F. Chang: Institute of High Energy Physics, Beijing.

L. C. Chang: Institute of Physics, National Chiao-Tung University, Hsinchu.

Y. Chang: National United University, Miao-Li.

H. S. Chen: Institute of High Energy Physics, Beijing.

Q. Y. Chen: Shandong University, Jinan.

S. M. Chen: Department of Engineering Physics, Tsinghua University, Beijing.

Y. X. Chen: North China Electric Power University, Beijing.

Y. Chen: Shenzhen University, Shenzhen.

J.-H. Cheng: Institute of Physics, National Chiao-Tung University, Hsinchu.

J. Cheng: Shandong University, Jinan.

Y. P. Cheng: Institute of High Energy Physics, Beijing.

Z. K. Cheng: Sun Yat-Sen (Zhongshan) University, Guangzhou.

J. J. Cherwinka: University of Wisconsin, Madison, WI 53706, USA.

M. C. Chu: Chinese University of Hong Kong, Hong Kong.

A. Chukanov: Joint Institute for Nuclear Research, Dubna, Moscow Region, Russia.

J. P. Cummings: Siena College, Loudonville, NY 12211, USA.

J. de Arcos: Department of Physics, Illinois Institute of Technology, Chicago, IL 60616, USA.

Z. Y. Deng: Institute of High Energy Physics, Beijing.

X. F. Ding: Institute of High Energy Physics, Beijing.

Y. Y. Ding: Institute of High Energy Physics, Beijing.

M. V. Diwan: Brookhaven National Laboratory, Upton, NY 11973, USA.

M. Dolgareva: Joint Institute for Nuclear Research, Dubna, Moscow Region, Russia.

J. Dove: Department of Physics, University of Illinois at Urbana-Champaign, Urbana, IL 61801, USA.

D. A. Dwyer: Lawrence Berkeley National Laboratory, Berkeley, CA 94720, USA.

W. R. Edwards: Lawrence Berkeley National Laboratory, Berkeley, CA 94720, USA.

R. Gill: Brookhaven National Laboratory, Upton, NY 11973, USA.

M. Gonchar: Joint Institute for Nuclear Research, Dubna, Moscow Region, Russia.

G. H. Gong: Department of Engineering Physics, Tsinghua University, Beijing.

H. Gong: Department of Engineering Physics, Tsinghua University, Beijing.

M. Grassi: Institute of High Energy Physics, Beijing.

W. Q. Gu: Department of Physics and Astronomy, Shanghai Jiao Tong University, Shanghai Laboratory for Particle Physics and Cosmology, Shanghai.

M. Y. Guan: Institute of High Energy Physics, Beijing.

L. Guo: Department of Engineering Physics, Tsinghua University, Beijing.

X. H. Guo: Beijing Normal University, Beijing.

Z. Guo: Department of Engineering Physics, Tsinghua University, Beijing.

R. W. Hackenburg: Brookhaven National Laboratory, Upton, NY 11973, USA.

R. Han: North China Electric Power University, Beijing.

S. Hans: Brookhaven National Laboratory, Upton, NY 11973, USA; Department of Chemistry and Chemical Technology, Bronx Community College, Bronx, NY 10453, USA.

M. He: Institute of High Energy Physics, Beijing.

K. M. Heeger: Department of Physics, Yale University, New Haven, CT 06520, USA.

Y. K. Heng: Institute of High Energy Physics, Beijing. 
A. Higuera: Department of Physics, University of Houston, Houston, TX 77204, USA.

Y. K. Hor: Center for Neutrino Physics, Virginia Tech, Blacksburg, VA 24061, USA.

Y. B. Hsiung: Department of Physics, National Taiwan University, Taipei.

B. Z. Hu: Department of Physics, National Taiwan University, Taipei.

T. Hu: Institute of High Energy Physics, Beijing.

W. Hu: Institute of High Energy Physics, Beijing.

E. C. Huang: Department of Physics, University of Illinois at Urbana-Champaign, Urbana, IL 61801, USA.

H. X. Huang: China Institute of Atomic Energy, Beijing.

X. T. Huang: Shandong University, Jinan.

P. Huber: Center for Neutrino Physics, Virginia Tech, Blacksburg, VA 24061, USA.

W. Huo: University of Science and Technology of China, Hefei.

G. Hussain: Department of Engineering Physics, Tsinghua University, Beijing.

D. E. Jaffe: Brookhaven National Laboratory, Upton, NY 11973, USA.

P. Jaffke: Center for Neutrino Physics, Virginia Tech, Blacksburg, VA 24061, USA.

K. L. Jen: Institute of Physics, National Chiao-Tung University, Hsinchu.

S. Jetter: Institute of High Energy Physics, Beijing.

X. P. Ji: School of Physics, Nankai University, Tianjin; Department of Engineering Physics, Tsinghua University, Beijing.

X. L. Ji: Institute of High Energy Physics, Beijing.

J. B. Jiao: Shandong University, Jinan.

R. A. Johnson: Department of Physics, University of Cincinnati, Cincinnati, OH 45221, USA.

D. Jones: Department of Physics, College of Science and Technology, Temple University, Philadelphia, PA 19122, USA.

J. Joshi: Brookhaven National Laboratory, Upton, NY 11973, USA.

L. Kang: Dongguan University of Technology, Dongguan.

S. H. Kettell: Brookhaven National Laboratory, Upton, NY 11973, USA.

S. Kohn: Department of Physics, University of California, Berkeley, CA 94720, USA.

M. Kramer: Lawrence Berkeley National Laboratory, Berkeley, CA 94720, USA; Department of Physics, University of California, Berkeley, CA 94720, USA.

K. K. Kwan: Chinese University of Hong Kong, Hong Kong.

M. W. Kwok: Chinese University of Hong Kong, Hong Kong.

T. Kwok: Department of Physics, The University of Hong Kong, Pokfulam, Hong Kong.

T. J. Langford: Department of Physics, Yale University, New Haven, CT 06520, USA.

K. Lau: Department of Physics, University of Houston, Houston, TX 77204, USA.

L. Lebanowski: Department of Engineering Physics, Tsinghua University, Beijing.

J. Lee: Lawrence Berkeley National Laboratory, Berkeley, CA 94720, USA.

J. H. C. Lee: Department of Physics, The University of Hong Kong, Pokfulam, Hong Kong.

R. T. Lei: Dongguan University of Technology, Dongguan.

R. Leitner: Faculty of Mathematics and Physics, Charles University, Prague, Czech Republic.

C. Li: Shandong University, Jinan.

D. J. Li: University of Science and Technology of China, Hefei.

F. Li: Institute of High Energy Physics, Beijing.

G. S. Li: Department of Physics and Astronomy, Shanghai Jiao Tong University, Shanghai Laboratory for Particle Physics and Cosmology, Shanghai.

Q. J. Li: Institute of High Energy Physics, Beijing.

S. Li: Dongguan University of Technology, Dongguan.

S. C. Li: Department of Physics, The University of Hong Kong, Pokfulam, Hong Kong; Center for Neutrino Physics, Virginia Tech, Blacksburg, VA 24061, USA.

W. D. Li: Institute of High Energy Physics, Beijing.

X. N. Li: Institute of High Energy Physics, Beijing.

Y. F. Li: Institute of High Energy Physics, Beijing.

Z. B. Li: Sun Yat-Sen (Zhongshan) University, Guangzhou.

H. Liang: University of Science and Technology of China, Hefei.

C. J. Lin: Lawrence Berkeley National Laboratory, Berkeley, CA 94720, USA.

G. L. Lin: Institute of Physics, National Chiao-Tung University, Hsinchu.

S. Lin: Dongguan University of Technology, Dongguan.

S. K. Lin: Department of Physics, University of Houston, Houston, TX 77204, USA.

Y.-C. Lin: Department of Physics, National Taiwan University, Taipei.

J. J. Ling: Sun Yat-Sen (Zhongshan) University, Guangzhou.

J. M. Link: Center for Neutrino Physics, Virginia Tech, Blacksburg, VA 24061, USA.

L. Littenberg: Brookhaven National Laboratory, Upton, NY 11973, USA.

B. R. Littlejohn: Department of Physics, Illinois Institute of Technology, Chicago, IL 60616, USA.

D. W. Liu: Department of Physics, University of Houston, Houston, TX 77204, USA.

J. L. Liu: Department of Physics and Astronomy, Shanghai Jiao Tong University, Shanghai Laboratory for Particle Physics and Cosmology, Shanghai.

J. C. Liu: Institute of High Energy Physics, Beijing.

C. W. Loh: Nanjing University, Nanjing.

C. Lu: Joseph Henry Laboratories, Princeton University, Princeton, NJ 08544, USA.

H. Q. Lu: Institute of High Energy Physics, Beijing.

J. S. Lu: Institute of High Energy Physics, Beijing. 
K. B. Luk: Department of Physics, University of California, Berkeley, CA 94720, USA; Lawrence Berkeley National Laboratory, Berkeley, CA 94720, USA.

Z. Lv: Department of Nuclear Science and Technology, School of Energy and Power Engineering, Xi'an Jiaotong University, Xi'an.

Q. M. Ma: Institute of High Energy Physics, Beijing.

X. Y. Ma: Institute of High Energy Physics, Beijing.

X. B. Ma: North China Electric Power University, Beijing.

Y. Q. Ma: Institute of High Energy Physics, Beijing.

Y. Malyshkin: Instituto de Física, Pontificia Universidad Católica de Chile, Santiago, Chile.

D. A. Martinez Caicedo: Department of Physics, Illinois Institute of Technology, Chicago, IL 60616, USA.

R. D. McKeown: California Institute of Technology, Pasadena, CA 91125, USA; College of William and Mary, Williamsburg, VA 23187, USA.

I. Mitchell: Department of Physics, University of Houston, Houston, TX 77204, USA.

M. Mooney: Brookhaven National Laboratory, Upton, NY 11973, USA.

Y. Nakajima: Lawrence Berkeley National Laboratory, Berkeley, CA 94720, USA.

J. Napolitano: Department of Physics, College of Science and Technology, Temple University, Philadelphia, PA 19122, USA.

D. Naumov: Joint Institute for Nuclear Research, Dubna, Moscow Region, Russia.

E. Naumova: Joint Institute for Nuclear Research, Dubna, Moscow Region, Russia.

H. Y. Ngai: Department of Physics, The University of Hong Kong, Pokfulam, Hong Kong.

Z. Ning: Institute of High Energy Physics, Beijing.

J. P. Ochoa-Ricoux: Instituto de Física, Pontificia Universidad Católica de Chile, Santiago, Chile.

A. Olshevskiy: Joint Institute for Nuclear Research, Dubna, Moscow Region, Russia.

H.-R. Pan: Department of Physics, National Taiwan University, Taipei.

J. Park: Center for Neutrino Physics, Virginia Tech, Blacksburg, VA 24061, USA.

S. Patton: Lawrence Berkeley National Laboratory, Berkeley, CA 94720, USA.

V. Pec: Faculty of Mathematics and Physics, Charles University, Prague, Czech Republic.

J. C. Peng: Department of Physics, University of Illinois at Urbana-Champaign, Urbana, IL 61801, USA.

L. Pinsky: Department of Physics, University of Houston, Houston, TX 77204, USA.

C. S. J. Pun: Department of Physics, The University of Hong Kong, Pokfulam, Hong Kong.

F. Z. Qi: Institute of High Energy Physics, Beijing.

M. Qi: Nanjing University, Nanjing.

X. Qian: Brookhaven National Laboratory, Upton, NY 11973, USA.

N. Raper: Department of Physics, Applied Physics, and Astronomy, Rensselaer Polytechnic Institute, Troy, NY 12180, USA.

J. Ren: China Institute of Atomic Energy, Beijing.

R. Rosero: Brookhaven National Laboratory, Upton, NY 11973, USA.

B. Roskovec: Faculty of Mathematics and Physics, Charles University, Prague, Czech Republic; Instituto de Física, Pontificia Universidad Católica de Chile, Santiago, Chile.

X. C. Ruan: China Institute of Atomic Energy, Beijing.

H. Steiner: Department of Physics, University of California, Berkeley, CA 94720, USA; Lawrence Berkeley National Laboratory, Berkeley, CA 94720, USA.

G. X. Sun: Institute of High Energy Physics, Beijing.

J. L. Sun: China General Nuclear Power Group, Shenzhen.

W. Tang: Brookhaven National Laboratory, Upton, NY 11973, USA.

D. Taychenachev: Joint Institute for Nuclear Research, Dubna, Moscow Region, Russia.

K. Treskov: Joint Institute for Nuclear Research, Dubna, Moscow Region, Russia.

K. V. Tsang: Lawrence Berkeley National Laboratory, Berkeley, CA 94720, USA.

C. E. Tull: Lawrence Berkeley National Laboratory, Berkeley, CA 94720, USA.

N. Viaux: Instituto de Física, Pontificia Universidad Católica de Chile, Santiago, Chile.

B. Viren: Brookhaven National Laboratory, Upton, NY 11973, USA.

V. Vorobel: Faculty of Mathematics and Physics, Charles University, Prague, Czech Republic.

C. H. Wang: National United University, Miao-Li.

M. Wang: Shandong University, Jinan.

N. Y. Wang: Beijing Normal University, Beijing.

R. G. Wang: Institute of High Energy Physics, Beijing.

W. Wang: Sun Yat-Sen (Zhongshan) University, Guangzhou; College of William and Mary, Williamsburg, VA 23187, USA.

X. Wang: College of Electronic Science and Engineering, National University of Defense Technology, Changsha.

Y. F. Wang: Institute of High Energy Physics, Beijing.

Z. Wang: Department of Engineering Physics, Tsinghua University, Beijing.

Z. Wang: Institute of High Energy Physics, Beijing.

Z. M. Wang: Institute of High Energy Physics, Beijing.

H. Y. Wei: Department of Engineering Physics, Tsinghua University, Beijing.

L. J. Wen: Institute of High Energy Physics, Beijing.

K. Whisnant: Iowa State University, Ames, IA 50011, USA.

C. G. White: Department of Physics, Illinois Institute of Technology, Chicago, IL 60616, USA.

L. Whitehead: Department of Physics, University of Houston, Houston, TX 77204, USA.

T. Wise: Department of Physics, Yale University, New Haven, CT 06520, USA.

H. L. H. Wong: Department of Physics, University of California, Berkeley, CA 94720, USA; Lawrence Berkeley National Laboratory, Berkeley, CA 94720, USA. 
S. C. F. Wong: Sun Yat-Sen (Zhongshan) University, Guangzhou.

E. Worcester: Brookhaven National Laboratory, Upton, NY 11973, USA.

C.-H. Wu: Institute of Physics, National Chiao-Tung University, Hsinchu.

Q. Wu: Shandong University, Jinan.

W. J. Wu: Institute of High Energy Physics, Beijing.

D. M. Xia: Chongqing University, Chongqing.

J. K. Xia: Institute of High Energy Physics, Beijing.

Z. Z. Xing: Institute of High Energy Physics, Beijing.

J. Y. Xu: Chinese University of Hong Kong, Hong Kong.

J. L. Xu: Institute of High Energy Physics, Beijing.

Y. Xu: Sun Yat-Sen (Zhongshan) University, Guangzhou.

T. Xue: Department of Engineering Physics, Tsinghua University, Beijing.

C. G. Yang: Institute of High Energy Physics, Beijing.

H. Yang: Nanjing University, Nanjing.

L. Yang: Dongguan University of Technology, Dongguan.

M. S. Yang: Institute of High Energy Physics, Beijing.

M. T. Yang: Shandong University, Jinan.

M. Ye: Institute of High Energy Physics, Beijing.

Z. Ye: Department of Physics, University of Houston, Houston, TX 77204, USA.

M. Yeh: Brookhaven National Laboratory, Upton, NY 11973, USA.

B. L. Young: Iowa State University, Ames, IA 50011, USA.

Z. Y. Yu: Institute of High Energy Physics, Beijing.

S. Zeng: Institute of High Energy Physics, Beijing.

L. Zhan: Institute of High Energy Physics, Beijing.

C. Zhang: Brookhaven National Laboratory, Upton, NY 11973, USA.

H. H. Zhang: Sun Yat-Sen (Zhongshan) University, Guangzhou.

J. W. Zhang: Institute of High Energy Physics, Beijing.

Q. M. Zhang: Department of Nuclear Science and Technology, School of Energy and Power Engineering, Xi' an Jiaotong University, Xi'an.

X. T. Zhang: Institute of High Energy Physics, Beijing.

Y. M. Zhang: Department of Engineering Physics, Tsinghua University, Beijing.

Y. X. Zhang: China General Nuclear Power Group, Shenzhen.

Y. M. Zhang: Sun Yat-Sen (Zhongshan) University, Guangzhou.

Z. J. Zhang: Dongguan University of Technology, Dongguan.

Z. Y. Zhang: Institute of High Energy Physics, Beijing.

Z. P. Zhang: University of Science and Technology of China, Hefei.

J. Zhao: Institute of High Energy Physics, Beijing.

Q. W. Zhao: Institute of High Energy Physics, Beijing.

Y. B. Zhao: Institute of High Energy Physics, Beijing.

W. L. Zhong: Institute of High Energy Physics, Beijing.

L. Zhou: Institute of High Energy Physics, Beijing.

N. Zhou: University of Science and Technology of China, Hefei.

H. L. Zhuang: Institute of High Energy Physics, Beijing.

J. H. Zou: Institute of High Energy Physics, Beijing.

Open Access This article is distributed under the terms of the Creative Commons Attribution 4.0 International License (http://creativecomm ons.org/licenses/by/4.0/), which permits unrestricted use, distribution, and reproduction in any medium, provided you give appropriate credit to the original author(s) and the source, provide a link to the Creative Commons license, and indicate if changes were made.

Funded by SCOAP ${ }^{3}$. 


\section{References}

1. B. Pontecorvo, Mesonium and anti-mesonium. Sov. Phys. JETP 6, 429 (1957). [Zh. Eksp. Teor. Fiz. 33, 549 (1957)]

2. B. Pontecorvo, Inverse beta processes and nonconservation of lepton charge. Sov. Phys. JETP 7, 172-173 (1958). [Zh. Eksp. Teor. Fiz. 34, 247 (1957)]

3. B.T. Cleveland et al., Measurement of the solar electron neutrino flux with the Homestake chlorine detector. Astrophys. J. 496, 505526 (1998). doi:10.1086/305343

4. F. Kaether et al., Reanalysis of the GALLEX solar neutrino flux and source experiments. Phys. Lett. B 685, 47-54 (2010). doi:10. 1016/j.physletb.2010.01.030. arXiv:1001.2731 [hep-ex]

5. J.N. Abdurashitov et al., Measurement of the solar neutrino capture rate with gallium metal. III: Results for the 2002-2007 data-taking period. Phys. Rev. C 80, 015807 (2009). doi:10.1103/PhysRevC. 80.015807. arXiv:0901.2200 [nucl-ex]

6. Y. Fukuda et al., Evidence for oscillation of atmospheric neutrinos. Phys. Rev. Lett. 81, 1562-1567 (1998). doi:10.1103/PhysRevLett. 81.1562

7. P. Adamson et al., Combined analysis of $v_{\mu}$ disappearance and $v_{\mu} \rightarrow v_{e}$ appearance in MINOS using accelerator and atmospheric neutrinos. Phys. Rev. Lett. 112, 191801 (2014). doi:10. 1103/PhysRevLett.112.191801

8. M.H. Ahn et al., Indications of neutrino oscillation in a $250 \mathrm{~km}$ long baseline experiment. Phys. Rev. Lett. 90, 041801 (2003). doi:10. 1103/PhysRevLett.90.041801

9. S. Abe et al., Precision measurement of neutrino oscillation parameters with KamLAND. Phys. Rev. Lett. 100, 221803 (2008). doi:10. 1103/PhysRevLett.100.221803. arXiv:0801.4589 [hep-ex]

10. F.P. An et al., Observation of electron-antineutrino disappearance at Daya Bay. Phys. Rev. Lett. 108, 171803 (2012). doi:10.1103/ PhysRevLett.108.171803. arXiv:1203.1669 [hep-ex]

11. J. Ahn et al., Phys. Rev. Lett. 108, 191802 (2012)

12. Y. Abe et al., Reactor electron antineutrino disappearance in the Double Chooz experiment. Phys. Rev. D 86, 052008 (2012). doi:10. 1103/PhysRevD.86.052008. arXiv:1207.6632 [hep-ex]

13. S. Eliezer, R.S. Arthur, Experimental consequences of electron neutrino-muon-neutrino mixing in neutrino beams. Nucl. Phys. B 105, 45-51 (1976). doi:10.1016/0550-3213(76)90059-6

14. H. Fritzsch, P. Minkowski, Vector-like weak currents, massive neutrinos, and neutrino beam oscillations. Phys. Lett. B 62, 72-76 (1976). doi:10.1016/0370-2693(76)90051-4

15. S.M. Bilenky, B. Pontecorvo., Again on neutrino oscillations. Lett. Nuovo Cim. 17, 569 (1976). doi:10.1007/BF02746567

16. E.Kh. Akhmedov, A.Yu. Smirnov, Paradoxes of neutrino oscillations. Phys. Atom. Nucl. 72, 1363-1381 (2009). doi:10.1134/ S1063778809080122. arXiv:0905.1903 [hep-ph]

17. C. Giunti, Coherence and wave packets in neutrino oscillations. Found. Phys. Lett. 17, 103-124 (2004). doi:10.1023/B:FOPL. 0000019651.53280.31. arXiv:hep-ph/0302026

18. M. Beuthe, Oscillations of neutrinos and mesons in quantum field theory. Phys. Rep. 375, 105-218 (2003). doi:10.1016/ S0370-1573(02)00538-0. arXiv:hep-ph/0109119

19. C. Giunti, C.W. Kim, Fundamentals of Neutrino Physics and Astrophysics (Oxford University Press, Oxford, UK, 2007)

20. A.E. Bernardini, S. De Leo, An analytic approach to the wave packet formalism in oscillation phenomena. Phys. Rev. D 70, 053010 (2004). doi:10.1103/PhysRevD.70.053010. arXiv:hep-ph/0411134

21. S. Nussinov, Solar neutrinos and neutrino mixing. Phys. Lett. B 63, 201-203 (1976). doi:10.1016/0370-2693(76)90648-1

22. B. Kayser, On the quantum mechanics of neutrino oscillation. Phys. Rev. D 24, 110 (1981). doi:10.1103/PhysRevD.24.110
23. K. Kiers, S. Nussinov, N. Weiss, Coherence effects in neutrino oscillations. Phys. Rev. D 53, 537-547 (1996). doi:10.1103/ PhysRevD.53.537. arXiv:hep-ph/9506271

24. E. Akhmedov, D. Hernandez, A. Smirnov, Neutrino production coherence and oscillation experiments. JHEP 1204, 052 (2012). doi:10.1007/JHEP04(2012)052. arXiv:1201.4128 [hep-ph]

25. B. Kayser, J. Kopp, Testing the wave packet approach to neutrino oscillations in future experiments (2010). arXiv: 1005.4081 [hep$\mathrm{ph}]$

26. W. Grimus, P. Stockinger, Real oscillations of virtual neutrinos. Phys. Rev. D 54, 3414-3419 (1996). doi:10.1103/PhysRevD.54. 3414. arXiv:hep-ph/9603430

27. C.Y. Cardall, D.J.H. Chung, The MSW effect in quantum field theory. Phys. Rev. D 60, 073012 (1999). doi:10.1103/PhysRevD. 60.073012. arXiv:hep-ph/9904291

28. P. Stockinger, Introduction to a field-theoretical treatment of neutrino oscillations. Pramana 54, 203-214 (2000). doi:10.1007/ s12043-000-0017-1

29. M. Beuthe, Towards a unique formula for neutrino oscillations in vacuum. Phys. Rev. D 66, 013003 (2002). doi:10.1103/PhysRevD. 66.013003. arXiv: hep-ph/0202068

30. C. Giunti et al., On the treatment of neutrino oscillations without resort to weak eigenstates. Phys. Rev. D 48, 4310-4317 (1993). doi:10.1103/PhysRevD.48.4310. arXiv:hep-ph/9305276

31. E.Kh. Akhmedov, J. Kopp, Neutrino oscillations: quantum mechanics vs. quantum field theory. JHEP 1004, 008 (2010). doi:10.1007/JHEP04(2010)008, doi:10.1007/JHEP10(2013)052. arXiv: 1001.4815 [hep-ph]

32. D.V. Naumov, V.A. Naumov, A diagrammatic treatment of neutrino oscillations. J. Phys. G 37, 105014 (2010). doi:10.1088/ 0954-3899/37/10/105014. arXiv:1008.0306 [hep-ph]

33. B.J.P. Jones, Dynamical pion collapse and the coherence of conventional neutrino beams. Phys. Rev. D 91(5), 053002 (2015). doi:10. 1103/PhysRevD.91.053002. arXiv:1412.2264 [hep-ph]

34. E. Lisi, A. Marrone, D. Montanino, Probing possible decoherence effects in atmospheric neutrino oscillations. Phys. Rev. Lett. 85, 1166-1169 (2000). doi:10.1103/PhysRevLett.85.1166. arXiv:hep-ph/0002053

35. T. Araki et al., Measurement of neutrino oscillation with KamLAND: evidence of spectral distortion. Phys. Rev. Lett. 94, 081801 (2005). doi:10.1103/PhysRevLett.94.081801. arXiv:hep-ex/0406035

36. G. Barenboim et al., Quantum decoherence and neutrino data. Nucl. Phys. B 758, 90-111 (2006). doi:10.1016/j.nuclphysb.2006. 09.012. arXiv:hep-ph/0603028

37. P. Adamson et al., Measurement of neutrino oscillations with the MINOS detectors in the NuMI beam. Phys. Rev. Lett. 101, 131802 (2008). doi:10.1103/PhysRevLett.101.131802. arXiv:0806.2237 [hep-ex]

38. H. Kwon et al., Phys. Rev. D 24, 1097 (1981)

39. B. Ackhar et al., Nucl. Phys. B 434, 503 (1995)

40. G. Zacek et al., Neutrino oscillation experiments at the Gosgen nuclear power reactor. Phys. Rev. D 34, 2621-2636 (1986). doi:10. 1103/PhysRevD.34.2621

41. G.S. Vidyakin et al., Bounds on the neutrino oscillation parameters for reactor anti-neutrinos. Sov. Phys. JETP 71, 424-426 (1990)

42. F. Boehm et al., Final results from the Palo Verde neutrino oscillation experiment. Phys. Rev. D 64, 112001 (2001). doi:10.1103/ PhysRevD.64.112001. arXiv:hep-ex/0107009

43. M. Apollonio et al., Search for neutrino oscillations on a long baseline at the CHOOZ nuclear power station. Eur. Phys. J. C 27, 331 (2003). arXiv:hep-ex/0301017

44. A.S. Dighe, Q.Y. Liu, A.Yu. Smirnov, Coherence and the daynight asymmetry in the solar neutrino flux (1999). arXiv: hep$-\mathrm{ph} / 9903329$ 
45. D.V. Naumov, On the theory of wave packets. Phys. Part. Nucl. Lett. 10, 642-650 (2013). doi:10.1134/S1547477113070145. arXiv:1309.1717 [quant-ph]

46. A.E. Bernardini, M.M. Guzzo, F.R. Torres, Second-order corrections to neutrino two-flavor oscillation parameters in the wave packet approach. Eur. Phys. J. C 48, 613 (2006). doi:10.1140/epjc/ s10052-006-0032-6. arXiv:hep-ph/0612001

47. Y. Farzan, A.Yu. Smirnov, Coherence and oscillations of cosmic neutrinos. Nucl. Phys. B 805, 356-376 (2008). doi:10.1016/j. nuclphysb.2008.07.028. arXiv:0803.0495 [hep-ph]

48. V.A. Naumov, D.S. Shkirmanov, Extended Grimus-Stockinger theorem and inverse square law violation in quantum field theory. Eur. Phys. J. C 73(11), 2627 (2013). doi:10.1140/epjc/ s10052-013-2627-z. arXiv:1309.1011 [hep-ph]

49. F.P. An et al., New measurement of antineutrino oscillation with the full detector configuration at Daya Bay. Phys. Rev. Lett. 115(11), 111802 (2015). doi:10.1103/PhysRevLett.115.111802. arXiv:1505.03456 [hep-ex]

50. F.P. An et al., A side-by-side comparison of Daya Bay antineutrino detectors. Nucl. Instrum. Methods A 685, 78-97 (2012). doi:10. 1016/j.nima.2012.05.030. arXiv:1202.6181 [physics.ins-det]

51. F.P. An et al., The muon system of the Daya Bay Reactor antineutrino experiment. Nucl. Instrum. Methods A 773, 8-20 (2015). doi:10.1016/j.nima.2014.09.070
52. F.P. An et al., The detector system of the Daya Bay reactor neutrino experiment. Nucl. Instrum. Methods A 811, 133-161 (2016). doi:10.1016/j.nima.2015.11.144. arXiv:1508.03943 [physics.insdet]

53. P. Vogel, J.F. Beacom, Angular distribution of neutron inverse beta decay, $\bar{v}_{e}+p \rightarrow e^{+}+n$. Phys. Rev. D 60, 053003 (1999). doi:10. 1103/PhysRevD.60.053003. arXiv:hep-ph/9903554

54. G.J. Feldman, R.D. Cousins, A unified approach to the classical statistical analysis of small signals. Phys. Rev. D 57, 3873-3889 (1998). doi:10.1103/PhysRevD.57.3873. arXiv:physics/9711021 [physics.data-an]

55. Cowan, G. et al. Asymptotic formulae for likelihood-based tests of new physics. Eur. Phys. J. C 71, 1554 (2011). doi:10.1140/ epjc/s10052-011-1554-0, doi:10.1140/epjc/s10052-013-2501-z. arXiv:1007.1727 [physics.data-an]. [Erratum: Eur. Phys. J. C 73, 2501 (2013)]

56. Z. Djurcic et al., JUNO conceptual design report (2015). arXiv:1508.07166 [physics.ins-det]

57. S.-B. Kim, New results from RENO and prospects with RENO50. Nucl. Part. Phys. Proc. 265-266, 93-98 (2015). doi:10.1016/j. nuclphysbps.2015.06.024. arXiv:1412.2199 [hep-ex] 